\title{
A intervenção motora como fator de prevenção de atrasos no desenvolvimento motor e cognitivo de bebês durante o período de internação hospitalar
}

\author{
Carolina Panceri ${ }^{\mathrm{a}}$, Keila Ruttnig Guidony Pereira ${ }^{\mathrm{b}}$, Nadia Cristina Valentini ${ }^{\mathrm{b}}$ \\ ${ }^{a}$ Hospital de Clínicas de Porto Alegre - HCPA, Porto Alegre, RS, Brasil. \\ ${ }^{b}$ Escola de Educação Física, Fisioterapia e Dança, Universidade Federal do Rio Grande do Sul - UFRGS, \\ Porto Alegre, RS, Brasil.
}

\begin{abstract}
Resumo: Introdução: A intervenção cognitivo-motora é benéfica para o desenvolvimento tanto motor como cognitivo do bebê. Essas intervenções, no hospital, têm sido amplamente estudadas dentro de unidades de terapia intensiva neonatal, porém poucos estudos investigam a intervenção avaliando o desenvolvimento infantil dentro de unidades de internação pediátrica. Objetivo: Avaliar o impacto da intervenção cognitivo-motora no desenvolvimento motor e cognitivo de bebês de 1 a 18 meses, hospitalizados por doenças respiratórias. Método: Pesquisa quase experimental, na qual participaram 22 bebês internados na unidade pediátrica por doença respiratória, divididos em dois grupos (10 no grupo controle e 12 no grupo intervenção), sem diferenças significativas nos dados biológicos e socioeconômicos. A idade variou de 1 a 16 meses, com média de 5,50 meses (DP \pm 4,51). Utilizou-se como instrumento um questionário para caracterização da amostra, a Alberta Infant Motor Scale (AIMS) e a Bayley Scales of Infant Development (BSID-III). A análise dos dados foi feita por meio de estatística descritiva, Teste $t$ de Student, General Linear Model e One Way ANOVA. Resultados: Os resultados evidenciaram interação significativa entre grupo $\times$ tempo, nos escores motores e cognitivos. Ao comparar os dois momentos, o grupo intervenção mudou positiva e significativamente do período pré para o pós-intervenção, nos escores motores e cognitivos. O mesmo não ocorreu para o grupo controle. Conclusão: Os resultados sugerem que a intervenção no ambiente hospitalar, durante o tempo de internação de bebês, contribui de forma positiva para o desenvolvimento motor e cognitivo.
\end{abstract}

Palavras-chave: Desenvolvimento Infantil, Fatores de Risco, Doenças Respiratórias, Intervenção Precoce.

\section{The motor intervention as delays prevention factor in motor and cognitive development of infants during the hospital stay}

\begin{abstract}
Introduction: Cognitive-motor tasks intervention is beneficial for the infant's motor and cognitive development. These interventions in the hospital setting, have been widely studied in neonatal intensive care units, however, few studies evaluate child development within pediatric units. Objective: To evaluate the impact of cognitive-motor intervention in motor and cognitive development of infants hospitalized with respiratory diseases. Method: The research was characterized as quasi-experimental, 22 babies hospitalized in the pediatric unit for respiratory disease were divided into 2 groups (10 in the control group and 12 in the intervention group) without significant differences in biological and socioeconomic data. The mean age was 5.50 months $(\mathrm{SD} \pm 4.51)$, ranging between 1 and 16 months. Questionnaire was conducted with the infant's parent/guardian for sample characterization. The Alberta Infant Motor Scale (AIMS) and the Bayley Scales of Infant Development (BSID-III) was used to evaluate motor e cognitive development. Data analysis was performed using descriptive statistics, Student's t test, General Linear Model and One Way ANOVA. Results: The results show a significant interaction between group
\end{abstract}

Autor para correspondência: Nadia Cristina Valentini, Universidade Federal do Rio Grande do Sul, Escola Superior de Educação Física, Rua Felizardo, 750, Bairro Jardim Botânico, CEP 90690-200, Porto Alegre, RS, Brasil, e-mail: nadiacv@esef.ufrgs.br

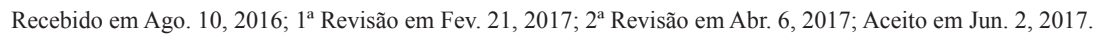


$\mathrm{x}$ time in motor and cognitive scores. When comparing the two times, the intervention group changed positively and significantly from pre- to post-intervention in motor and cognitive scores. The same was not observed for the control group. Conclusion: The results of this study suggest that the intervention during the hospital stay contributes positively to the motor and cognitive development.

Keywords: Child Development, Risk Factors, Respiratory Tract Diseases, Early Intervention.

\section{Introdução}

A intervenção precoce para bebês, caracterizada por programas de intervenção compensatória ou estimulação, é centrada principalmente em tarefas cognitivo-motoras, que permitem à criança mais oportunidades de interagir, experimentar e explorar o ambiente, facilitando o seu desenvolvimento global (BLAUW-HOSPERS; HADDERS-ALGRA, 2005; BLAUW-HOSPERS et al., 2007; WILLRICH; AZEVEDO; FERNANDES, 2009). Algumas pesquisas apontam que este tipo de intervenção é benéfico para o desenvolvimento motor do bebê (BLAUW-HOSPERS et al., 2007; ALMEIDA; VALENTINI, 2010; PEREIRA et al., 2011; SCHLITTLER et al., 2011), assim como para o desenvolvimento cognitivo (BLAUW-HOSPERS et al., 2007; ALMEIDA; VALENTINI, 2010). As experiências cognitivo-motoras formarão a base para o aprendizado de habilidades cognitivas da criança (WILLRICH; AZEVEDO; FERNANDES, 2009; HOLT; MIKATI, 2011).

Essas intervenções, no ambiente hospitalar, têm sido amplamente estudadas dentro de unidades de terapia intensiva (UTI) neonatal (ULLENHAG; PERSSON; NYQVIST, 2009; FERREIRA; BERGAMASCO, 2010). Arte-terapia, música, massagem e estimulação multissensorial são algumas das abordagens encontradas na literatura, avaliando-se diferentes parâmetros, como condiçôes clínicas, cardiorrespiratórias, nutricionais e de desenvolvimento de prematuros (ULLENHAG; PERSSON; NYQVIST, 2009; FERREIRA; BERGAMASCO, 2010; STANDLEY; SWEDBERG, 2011; VALLADARES; SILVA, 2011; SMITH et al., 2013). Porém, poucas pesquisas têm abordado esta temática fora da UTI neonatal.

A hospitalização, tanto em UTI neonatal como em unidades de internação pediátrica, tem sido apontada, em vários estudos, como um dos fatores de risco que influenciam o desenvolvimento dos bebês (HALPERN et al., 2000; HALPERN; FIGUEIRAS, 2004; GRANTHAM-MCGREGOR et al., 2007; MARIA-MENGEL; LINHARES, 2007; WALKER et al., 2007; AMORIM et al., 2009; WALKER et al., 2011; GABBARD; KREBS, 2012; PANCERI et al., 2012). A hospitalização na infância pode se configurar como uma experiência desagradável e traumática (MITRE; GOMES, 2004; MUNHÓZ; ORTIS, 2006; BERSCH; YUNES, 2008). O ambiente do hospital é novo, desconhecido e, muitas vezes, assustador para a criança (MITRE; GOMES, 2004; BERSCH; YUNES, 2008; OLIVEIRA; OLIVEIRA, 2008). Além da restriçâo de espaço físico, intensa iluminação, aparelhos, fios, estimulação sonora, com alarmes e ruídos estranhos, o bebê será manipulado por pessoas desconhecidas e será submetido a uma série de procedimentos, muitas vezes, dolorosos (MOTTA; ENUMO, 2004; ARAÚJO; RODRIGUES, 2010). Acrescenta-se ainda a necessidade de se afastar do convívio com familiares e pessoas mais próximas, uma vez que não é permitida a permanência de todos os familiares junto ao leito hospitalar (MITRE; GOMES, 2004; MOTTA; ENUMO, 2004; MUNHÓZ; ORTIS, 2006; OLIVEIRA; OLIVEIRA, 2008). Este ambiente, aliado à doença enfrentada, promove um confronto com a dor, a inatividade e a passividade, gerando sentimentos de medo, angústia, culpa, ansiedade e irritabilidade, bem como sensaçáo de abandono (MITRE; GOMES, 2004; MUNHÓZ; ORTIZ, 2006).

Ambientes como o hospital, privados de estímulos adequados, podem interferir de forma negativa, acarretando alteraçôes ou atrasos no desenvolvimento global do bebê (WILLRICH; AZEVEDO; FERNANDES, 2009; PANCERI et al., 2012). Este cenário agrava a situação da própria doença, que, no caso das doenças respiratórias, já apresentam um possível risco para um atraso na função motora do bebê (GRAMINHA; MARTINS, 1997; NICACIO; RIBEIRO, 2010). O processo de acometimento do trato respiratório em lactentes implica em aumento do trabalho ventilatório, acarretando maior consumo energético, com a finalidade de suprir a demanda ventilatória (NICACIO; RIBEIRO, 2010). Como as crianças pequenas já possuem elevada taxa metabólica, o maior consumo energético e o elevado gasto calórico podem gerar falta de disposição necessária para participação em atividades facilitadoras para seu desenvolvimento (GRAMINHA; MARTINS, 1997; NICACIO; RIBEIRO, 2010).

Desta forma, bebês que necessitam de internação hospitalar por doenças respiratórias devem receber maior atenção em relação ao seu desenvolvimento. A detecção precoce de bebês em risco de atraso de 
desenvolvimento oferece a oportunidade de intervir o mais cedo possível (HALPERN et al., 2000; HALPERN; FIGUEIRAS, 2004; RESEGUE; PUCCINI; SILVA, 2008; AMORIM et al., 2009; WALKER et al., 2011). O desenvolvimento motor e cognitivo em bebês expostos a risco, assim como programas interventivos para maximizar o desempenho destes bebês, prevenir ou minimizar atrasos, tem sido foco de grande interesse na literatura. Porém, poucos estudos investigam programas de intervenção, avaliando-se o desenvolvimento infantil dentro de unidades de internação pediátrica (PANCERI et al., 2012).

Assim, o presente estudo teve como objetivo avaliar o impacto da intervençáo motora no desenvolvimento motor e cognitivo de bebês de 1 a 18 meses, hospitalizados por doenças respiratórias. As seguintes hipóteses foram estabelecidas: (1) Bebês do grupo intervenção (GI) apresentarão mudanças positivas e significativas no desempenho motor e cognitivo do período pré para o pós-intervenção, enquanto que, para o grupo controle (GC), essas mudanças não são esperadas; (2) o GI apresentará desempenho motor e cognitivo superior no pós-intervenção, quando comparado ao GC.

\section{Método}

Este estudo se caracteriza como quase experimental (THOMAS; NELSON; SILVERMAN, 2012).

\subsection{Delineamento e participantes}

Este estudo foi realizado na unidade de internação pediátrica de um hospital público do sul do Brasil. O estudo foi aprovado pelo Comitê de Ética do Hospital de Clínicas de Porto Alegre (processo n. ${ }^{\circ}$ 13-0470), seguindo as normas estabelecidas pela Resolução no 466/12 do Conselho Nacional de Saúde, que regulamenta a pesquisa com seres humanos. Foram obtidas autorizações por meio de Termo de Consentimento Livre e Esclarecido dos responsáveis por cada bebê.

Inicialmente, foram convidados e aceitaram participar do estudo, os respectivos responsáveis por 39 bebês, que preencheram os seguintes critérios de inclusão: (1) faixa etária de 1 a 18 meses; (2) hospitalização por motivo de doenças respiratórias, conforme diagnóstico médico, e (3) ausência de sinais e sintomas de doenças neurológicas ou mentais previamente diagnosticadas ou doenças que poderiam ser fator interveniente nos resultados e delimitadores na avaliação. Destes, 17 não realizaram a segunda avaliaçấo pelos motivos de alta hospitalar não programada e sem o prévio conhecimento da pesquisadora; sono ou indisposição do bebê no dia da alta. Desta forma, a amostra deste estudo foi composta por 22 bebês, sendo 11 (50\%) do sexo masculino e 11 (50\%) do sexo feminino, com idade média de 5,50 meses $(\mathrm{DP} \pm 4,51)$ de idade, variando entre 1 e 16 meses. Em relação à idade gestacional, apenas dois (9,09\%) bebês eram prematuros, enquanto $20(90,91 \%)$ nasceram a termo. O peso ao nascer teve média de $2,872 \mathrm{~kg}$ $( \pm 0,536)$ e o comprimento de $46,23 \mathrm{~cm}( \pm 3,891)$. Todas as características biológicas e socioeconômicas das crianças foram analisadas e observa-se uma amostra semelhante, sem diferenças significativas nos dados disponibilizados.

\subsection{Procedimentos}

A pesquisadora monitorou as baixas e altas hospitalares através do sistema de prontuários eletrônicos, pelo período de oito meses. O prontuário on-line do paciente foi acessado para verificar o diagnóstico e as informaçóes que os responsáveis não souberam informar. Os pacientes de 1 a 18 meses que preencheram os critérios de inclusão foram convidados de forma consecutiva para participar $\mathrm{da}$ pesquisa, através do contato da pesquisadora com os pais ou responsáveis legais pela criança.

Os instrumentos para avaliação do desenvolvimento foram aplicados em dois momentos: após a baixa hospitalar, assim que os pacientes demonstravam-se estáveis, e quando os mesmos estavam com alta programada. Estas avaliaçóes foram realizadas na sala de recreação da unidade, nos horários fora de seu funcionamento, ou no próprio leito, garantindo assim um ambiente tranquilo e apropriado. Todos os testes foram filmados e posteriormente analisados por dois examinadores independentes, cujas avaliações apresentaram elevado valor de concordância (ICC > 85), sem diferenças significativas (testes de Friedman e Wilcoxon $-\mathrm{p}>0,5$ ).

Os pacientes que preencheram os critérios de inclusão e aceitaram participar da pesquisa foram divididos em dois grupos: grupo controle (GC) e grupo interventivo (GI). A divisão foi realizada através de sorteio aleatório. Se os pais das crianças não sorteadas desejassem participar, o acesso à intervenção era garantido.

Para caracterização da amostra, foi aplicado um questionário aos responsáveis, abordando os aspectos pré, peri e pós-natais dos bebês, como data de nascimento; idade gestacional; índice de Apgar; peso, comprimento e perímetro cefálico ao nascer; período de internação em UTI neonatal e dias em 
ventilação mecânica; período desta internação e dias que permaneceu em UTI pediátrica, além de dados relativos ao diagnóstico médico e à renda familiar.

A Alberta Infant Motor Scale (AIMS) foi utilizada para avaliar o desenvolvimento motor dos bebês. A facilidade de aplicação da AIMS, o baixo custo e os valores satisfatórios de validade e confiabilidade, demonstrados em vários estudos, fazem desta escala um instrumento de grande utilidade tanto no acompanhamento do desenvolvimento motor de bebês de 0 a 18 meses como na sua utilização em pesquisas científicas (ALMEIDA et al., 2008).

A AIMS é um instrumento basicamente de observação, desenvolvido no Canadá, criado para avaliar o desenvolvimento motor de bebês de 0 a 18 meses de idade. Composta por 58 itens, agrupados em quatro subescalas, que correspondem a quatro posições básicas: prono (21 itens), supino (9 itens), sentado (12 itens) e em pé (16 itens). Durante a avaliação, o examinador observa a livre movimentação da criança em cada uma das posições, levando em consideraçáo certos aspectos, tais como a superfície do corpo que sustenta o peso, a postura e os movimentos antigravitacionais. Cada item observado no repertório das habilidades motoras da criança recebe escore 1 (um) e cada item não observado recebe escore 0 (zero). Os itens observados em cada uma das subescalas sáo somados, resultando em quatro subtotais; o escore total ( 0 - 58 pontos) resultará da soma destes subtotais. Este é convertido em percentil de desenvolvimento motor, seguindo os critérios de classificaçáo, segundo normativa: a) desempenho motor normal/esperado: acima de $25 \%$ da curva percentílica; b) desempenho motor suspeito: entre $25 \%$ e $5 \%$ da curva percentílica; c) desempenho motor anormal: abaixo de $5 \%$ da curva percentílica (PIPER; DARRAH, 1994; SACCANI, 2009).

A Alberta Infant Motor Scale (AIMS) foi validada para a população brasileira e os resultados encontrados sugerem que a versão em português, Escala Motora Infantil de Alberta (EMIA), evidencia: (1) validade de conteúdo em termos de clareza $(\alpha=66,7$ a $\alpha=92,8)$ e pertinência (superiores a 0,98 ); (2) índices de teste-reteste confiáveis, sem alteraçóes significativas entre os dois momentos e com ótima confiabilidade $(\alpha=0,88)$ no geral e nas posturas (prono, $\alpha=0,86$; supino, $\alpha=0,89$; sentado, $\alpha=0,80$ e em pé, $\alpha=0,85$ ), além da capacidade discriminante para o grupo a termo e pré-termo $(-4,842 ; \mathrm{p} \leq 0,001)($ SACCANI, 2009; VALENTINI; SACCANI, 2012).

Para avaliar o desenvolvimento cognitivo e motor dos bebês, foi utilizada a escala da Bayley Scales of Infant Development (BSID-III). A escala cognitiva avalia aspectos relacionados ao desenvolvimento sensório-motor, como exploração, manipulação, relacionamento com objetos e formação de conceito de crianças entre 0 e 42 meses de idade. A pontuaçáo do desempenho é feita de acordo com a idade, sendo o escore bruto obtido através da soma dos itens, acrescido da soma dos itens das idades anteriores 18 .

A Escala Bayley é uma escala americana que avalia o desenvolvimento cognitivo, motor, socioemocional, do comportamento adaptativo e da linguagem de crianças entre 0 e 42 meses de idade. No presente estudo, utilizamos apenas as escalas que avaliam os domínios cognitivo e motor. A escala cognitiva avalia aspectos relacionados ao desenvolvimento sensório-motor, como exploração e manipulação, relacionamento de objetos, formação de conceito, entre outros aspectos do processamento cognitivo. A escala motora, dividida em subtestes motor fino e grosso, avalia preensão, integração perceptomotora, planejamento motor e velocidade motora (fino), além de movimentos dos membros e tronco, posicionamento estático (sentado, em pé, etc.), movimentos dinâmicos, incluindo locomoção e coordenação, equilíbrio e planejamento motor (grosso).

A BSID-III é padronizada, amplamente reconhecida na literatura, e possui uma amostra normativa constituída por 1.700 crianças representativas de toda a população dos Estados Unidos (BAYLEY, 1993). Está entre as melhores escalas existentes na área de avaliação do desenvolvimento infantil, fornecendo resultados confráveis, válidos e precisos (GABBARD; RODRIGUES, 2006; CAMPOS et al., 2006). Foi realizada a adaptação para a população brasileira apenas na faixa etária de 12 a 42 meses (MADASHI, 2012); no entanto, essa escala é amplamente utilizada em ambulatórios de seguimento e em pesquisas científicas da área de saúde da criança (CAMPOS et al., 2006).

A BSID-III pontua o desempenho da criança de acordo com sua idade e, para cada idade, há um ponto de início dos itens a serem administrados. O escore bruto é dado pela soma de todos os itens para os quais a criança recebeu crédito, acrescido da soma dos itens das idades anteriores. Através do escore bruto, é possível se obter o escore escalonado e o escore composto (escores padronizados). A classificação do desempenho da criança é feita através do escore composto: muito superior (>130), superior (120-129), médio alto (110-119), médio (90-109), médio baixo (80-89), limítrofe (70-79) e extremamente baixo $(<69)$. 


\subsection{Intervenção}

O programa de intervenção cognitivo-motora aconteceu diariamente, por 30 minutos, durante o tempo de internaçáo do bebê. As sessóes aconteceram no próprio leito ou na sala de recreação da unidade, conforme a liberação do controle de infecção hospitalar, e contou com a presença dos responsáveis, sempre que estes se encontravam no hospital. Cada sessão foi composta por atividades lúdicas, que oportunizam a experimentação ao bebê de novas habilidades motoras e cognitivas, da seguinte forma: 5 minutos iniciais destinados à ambientação do bebê; 5 minutos na posiçáo deitada - supino ou prono - com atividades de alongamentos, estímulo do rolar, perseguição visual e exploração de brinquedos; 10 minutos na posição sentada, enfatizando o controle postural e a manipulaçáo de objetos; e, por fim, 10 minutos favorecendo o deslocamento da criança, quer seja através do arrastar, engatinhar ou da marcha, com ou sem apoio. Esta sequência foi elaborada em ordem de progressão e com níveis de dificuldade adequados à faixa etária de cada bebê.

\subsection{Procedimentos para análise dos dados}

Os dados foram analisados utilizando-se o programa estatístico SPSS (versão 18.0). Foi realizada estatística descritiva com distribuição de frequência, medidas de tendência central e variabilidade. A comparação das características dos grupos foi feita por meio do Teste t de Student. Para avaliar o impacto da intervençáo motora no desenvolvimento cognitivo e motor das crianças investigadas, foi utilizado o General Linear Model, sendo adotado o critério Wilk's Lambda. Quando houve interação entre grupo e tempo, foram realizados testes post hoc para determinar em que aspecto se estabeleciam estas diferenças: Teste $t d e$ Student para dados pareados (comparação do pré para o pós, dentro dos grupos) e One Way ANOVA (comparação entre os grupos, nos dois momentos avaliativos). O nível de significância adotado foi $\mathrm{p} \leq 0,05$. Ainda, o valor de $p$ entre 0,05 e 0,08 foi considerado como tendência à significância.

\section{Resultados}

\subsection{Desempenho motor}

Os resultados evidenciaram uma interação significativa entre grupo $\times$ tempo nos escores motores da AIMS, nas posturas prono, supino e no escore total. As posturas sentado e em pé, assim como o percentil da AIMS e todos os escores motores da Bayley, não demonstraram interação significativa.

Ao comparar os dois momentos, o grupo intervenção mudou positiva e significativamente do período pré para o pós-intervenção, em todos os escores motores, com exceção do percentil da AIMS e do escore motor amplo escalado da Bayley: motor fino bruto $(p=0,003)$, motor fino escalado $(p=0,05)$, motor amplo bruto $(p=0,01)$, motor escalado $(\mathrm{p}=0,05)$, motor composto $(0,05)$, percentil motor Bayley $(\mathrm{p}=0,01)$, AIMS prono $(\mathrm{p}=0,03)$, AIMS supino $(\mathrm{p}=0,02)$, AIMS sentado $(\mathrm{p}=0,017)$, AIMS em pé $(\mathrm{p}=0,039)$, AIMS total $(\mathrm{p}=0,003)$. Tal comportamento não foi observado no grupo controle, em que nenhum dos escores motores teve mudanças positivas e significativas do momento pré para o pós-intervenção (Figuras 1, 2 e 3).

No fator grupo, não houve diferença significativa no pré, nem no pós-intervenção, em nenhum dos escores motores, conforme apresentado na Tabela 1 .

\subsection{Desempenho cognitivo}

Os resultados evidenciaram uma interação significativa entre grupo $\times$ tempo, nos escores cognitivo bruto, escalado e composto, comportamento não observado no percentil. Não houve diferença estatisticamente significativa entre os grupos no pré e pós-intervenção nos escores cognitivos, conforme apresentado na Tabela 1. No fator tempo, o grupo intervenção demonstrou mudanças positivas e significativas do período pré para o pós-intervenção no escore cognitivo bruto $(\mathrm{p}=0,006)$; os demais escores cognitivos apresentaram mudanças positivas e tendência à significância [escalado $(\mathrm{p}=0,067)$, composto $(\mathrm{p}=0,067)$ e percentil $(\mathrm{p}=0,077)]$. O mesmo não ocorreu para o grupo controle, em que nenhum dos escores cognitivos teve diferença significativa do momento pré para o pós-intervençáo, com valores de $p$ entre 0,193 e 0,358 (Figuras 3 e 4). Os resultados observados sugerem efeitos positivos da intervenção no desenvolvimento cognitivo de bebês hospitalizados.

\section{Discussão}

O presente estudo teve como objetivo avaliar o impacto da intervenção motora no desenvolvimento motor e cognitivo de bebês de 1 a 16 meses, hospitalizados por doenças respiratórias. De forma geral, a intervenção se mostrou benéfica ao processo desenvolvimental destes bebês, compensando, provavelmente, em parte, as situaçôes negativas vivenciadas no contexto da hospitalização. 


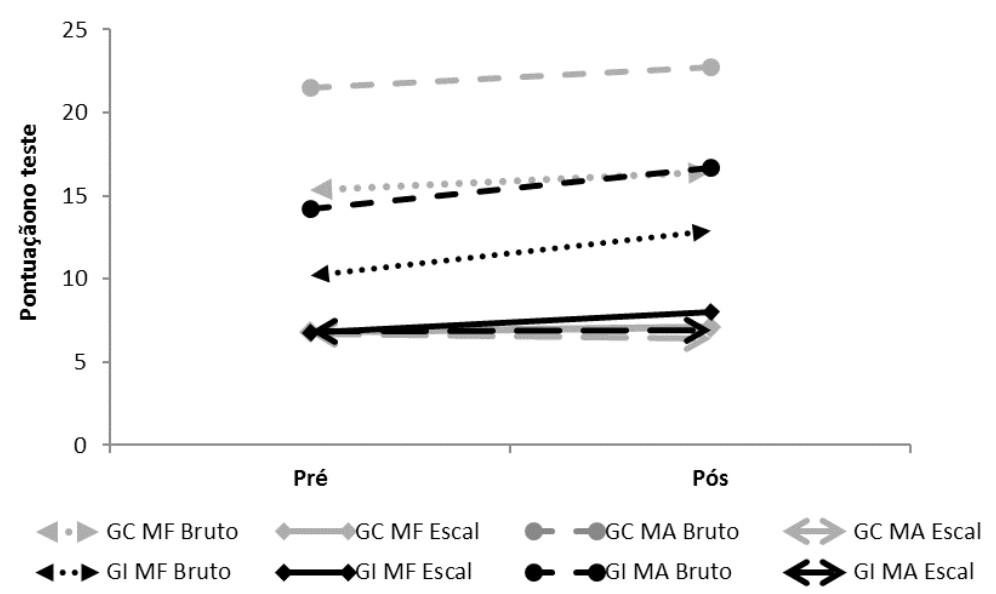

Figura 1. Comparação de médias do grupo controle (GC) e do grupo intervenção (GI), nos momentos pré e pós-intervenção, nos escores motor fino (MF) bruto e escalado, e motor amplo (MA) bruto e escalado da Bayley.

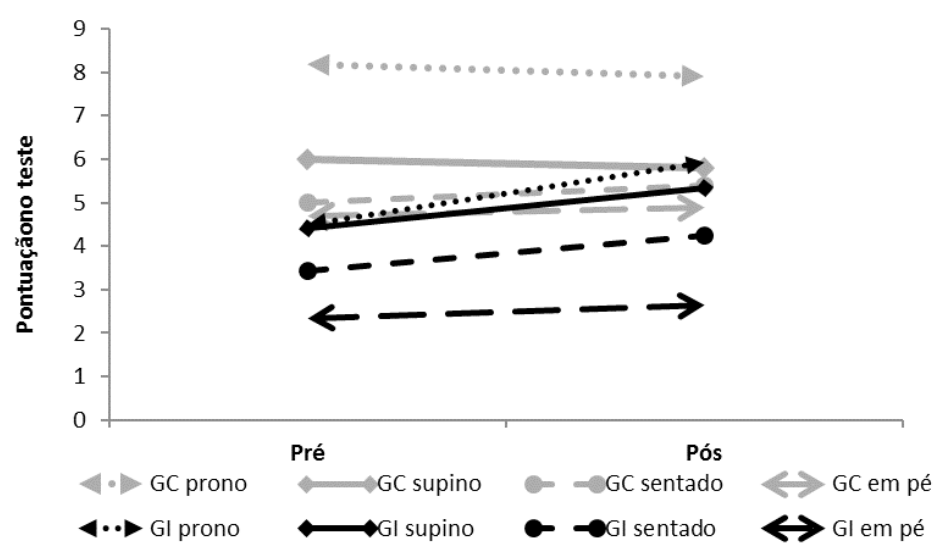

Figura 2. Comparação de médias do grupo controle (GC) e do grupo intervenção (GI), nos momentos pré e pós-intervenção, nos escores por postura da AIMS.

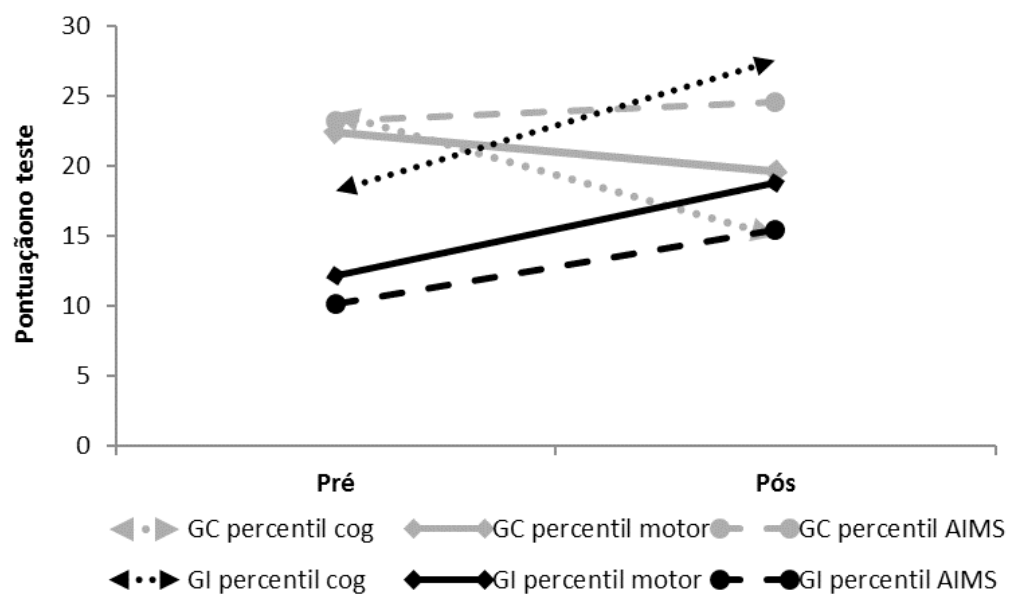

Figura 3. Comparação de médias do GC e GI nos momentos pré e pós-intervenção, nos percentis cognitivo e motor da Bayley, e percentil da AIMS. 


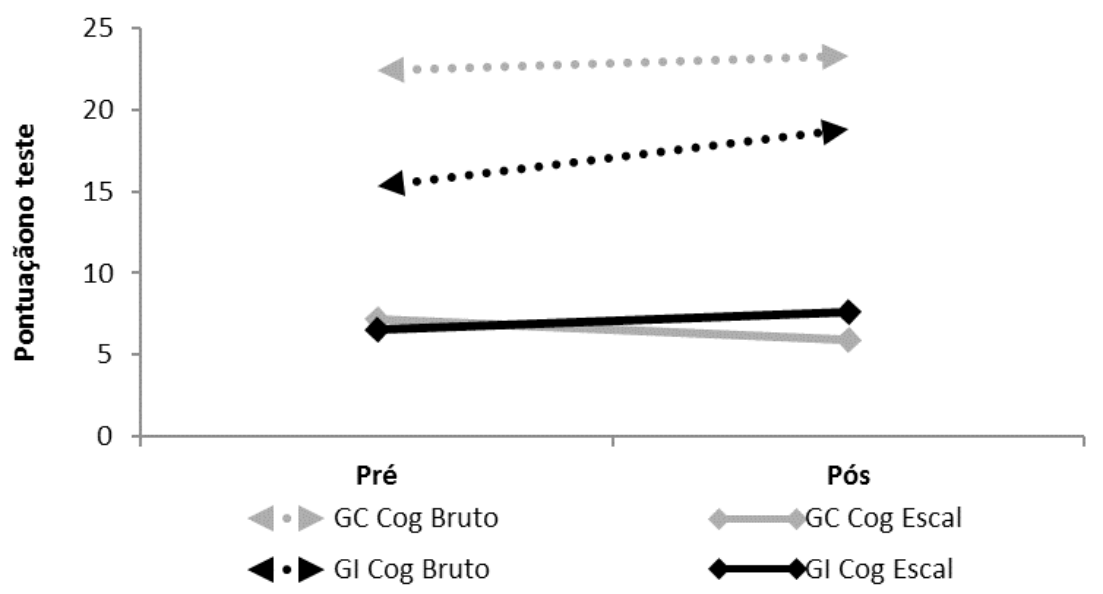

Figura 4. Comparação de médias do grupo controle (GC) e do grupo intervenção (GI), nos momentos pré e pós-intervenção, nos escores cognitivo bruto e cognitivo escalado da Bayley.

Tabela 1. Diferenças entre os grupos nos períodos pré e pós-intervenção.

\begin{tabular}{|c|c|c|c|c|c|c|}
\hline & \multicolumn{2}{|c|}{ Pré-intervenção } & \multirow{3}{*}{$p$} & \multicolumn{2}{|c|}{ Pós-intervenção } & \multirow{3}{*}{$p$} \\
\hline & GC & GI & & GC & GI & \\
\hline & Média \pm DP & Média \pm DP & & Média \pm DP & Média \pm DP & \\
\hline Cognitivo Bruto & $22,40 \pm 14,75$ & $15,33 \pm 11,46$ & 0,22 & $23,30 \pm 15,91$ & $18,83 \pm 11,22$ & 0,45 \\
\hline Cognitivo Escalado & $7,20 \pm 2,82$ & $6,58 \pm 2,35$ & 0,582 & $5,90 \pm 2,60$ & $7,58 \pm 2,81$ & 0,164 \\
\hline Cognitivo Composto & $86,00 \pm 14,10$ & $82,92 \pm 11,76$ & 0,582 & $79,50 \pm 13,00$ & $87,92 \pm 14,05$ & 0,164 \\
\hline Cognitivo Percentil & $23,80 \pm 26,46$ & $18,28 \pm 15,92$ & 0,552 & $15,04 \pm 17,07$ & $27,59 \pm 22,35$ & 0,161 \\
\hline Motor Fino Bruto & $15,30 \pm 10,20$ & $10,17 \pm 8,27$ & 0,207 & $16,40 \pm 10,74$ & $12,92 \pm 7,35$ & 0,379 \\
\hline Motor Fino Escalado & $6,80 \pm 3,61$ & $6,75 \pm 2,05$ & 0,968 & $7,10 \pm 3,17$ & $8,00 \pm 2,132$ & 0,438 \\
\hline Motor Amplo Bruto & $21,50 \pm 14,73$ & $14,17 \pm 11,07$ & 0,198 & $22,70 \pm 15,28$ & $16,67 \pm 10,73$ & 0,291 \\
\hline Motor Amplo Escalado & $6,70 \pm 3,05$ & $6,83 \pm 1,64$ & 0,897 & $6,40 \pm 2,75$ & $6,92 \pm 1,88$ & 0,608 \\
\hline Motor Escalado & $13,50 \pm 6,53$ & $13,58 \pm 2,27$ & 0,967 & $13,50 \pm 5,72$ & $14,92 \pm 3,11$ & 0,469 \\
\hline Motor Composto & $80,50 \pm 19,60$ & $80,75 \pm 6,82$ & 0,967 & $80,50 \pm 17,16$ & $84,75 \pm 9,35$ & 0,469 \\
\hline Motor Percentil & $22,42 \pm 22,30$ & $12,16 \pm 8,83$ & 0,158 & $19,64 \pm 20,45$ & $18,79 \pm 10,33$ & 0,901 \\
\hline AIMS prono & $8,20 \pm 7,11$ & $4,50 \pm 5,09$ & 0,171 & $7,90 \pm 7,43$ & $5,92 \pm 6,05$ & 0,498 \\
\hline AIMS supino & $6,00 \pm 3,30$ & $4,42 \pm 3,08$ & 0,259 & $5,80 \pm 3,15$ & $5,33 \pm 2,99$ & 0,726 \\
\hline AIMS sentado & $5,00 \pm 4,64$ & $3,42 \pm 3,67$ & 0,382 & $5,40 \pm 4,30$ & $4,25 \pm 3,91$ & 0,519 \\
\hline AIMS em pé & $4,70 \pm 5,98$ & $2,33 \pm 1,37$ & 0,197 & $4,90 \pm 5,87$ & $2,63 \pm 1,49$ & 0,217 \\
\hline AIMS total & $23,90 \pm 19,75$ & $14,67 \pm 12,36$ & 0,196 & $24,00 \pm 19,68$ & $18,17 \pm 13,80$ & 0,425 \\
\hline AIMS percentil & $23,21 \pm 27,87$ & $10,16 \pm 12,69$ & 0,161 & $24,61 \pm 35,38$ & $15,50 \pm 13,97$ & 0,421 \\
\hline
\end{tabular}

$\mathrm{Na}$ análise do desempenho motor e cognitivo, as crianças do GI evidenciaram mudanças positivas e significativas da pré para a pós-intervenção nos escores cognitivo e motor, confirmando a primeira hipótese da pesquisa. Isso vem ao encontro de estudos prévios que investigam efeitos da intervenção em crianças em risco para atrasos no desenvolvimento na mesma faixa etária (RAMEY; RAMEY, 1998; SANTOS-MONTEIRO et al., 2002; FORMIGA; PEDRAZZANI; TUDELLA, 2004;
BLAUW-HOSPERS et al., 2007; GRANTHAMMCGREGOR et al., 2007; BONNIER, 2008; ORTON et al., 2009; WILLRICH; AZEVEDO; FERNANDES, 2009; HOLT; MIKATI, 2011). Em um estudo de revisão sistemática, a eficácia da intervenção motora no desenvolvimento infantil é sugerida como benéfica. Os autores ressaltam a importância de o tipo de intervenção ser adequada para a idade da criança, especialmente para os prematuros (BLAUW-HOSPERS; HADDERS-ALGRA, 2005). 
No contexto da hospitalização infantil, grande atenção é dada à UTI neonatal, com diversos estudos interventivos, que utilizam variadas estratégias no manejo dos bebês internados, sugerindo efeitos positivos dessas práticas (ULLENHAG; PERSSON; NYQVIST, 2009; FERREIRA; BERGAMASCO, 2010; STANDLEY; SWEDBERG, 2011; VALLADARES; SILVA, 2011; SMITH et al., 2013). Por exemplo, um estudo que avaliou o impacto de um programa de intervenção com 109 mães e 123 bebês prematuros, durante o período de internação em UTI neonatal, em que as máes receberam um treinamento para proporcionar estimulação multissensorial, toque, movimento e massagem nos seus filhos. Aos 6 meses de idade corrigida, as crianças do grupo interventivo apresentaram escores significativamente mais altos sobre a comunicação e o comportamento simbólico (MILGROM et al., 2013). Outro estudo comparou o desempenho motor de 126 bebês internados em uma UTI neonatal antes e depois da implementação de um programa de intervenção, que incluía técnicas de posicionamento e suporte para autorregulação motora. Os bebês internados após a implementação do programa apresentaram maiores níveis de desenvolvimento motor (ULLENHAG; PERSSON; NYQVIST, 2009). A avaliação do impacto da intervenção tátil-cinestésica e multissensorial em bebês durante a internação em UTI neonatal demonstrou que os bebês do grupo experimental apresentaram melhor desempenho motor e mental (NELSON et al., 2001; FERREIRA; BERGAMASCO, 2010).

Os dados apresentados na presente pesquisa são diferenciais, pois trazem a intervenção motora fora da UTI, mas ainda dentro do ambiente hospitalar. Embora a literatura recente venha demonstrando a importância da intervençáo precoce nas mais diversas populaçóes, com ou sem risco para atrasos no desenvolvimento (BLAUW-HOSPERS; HADDERS-ALGRA, 2005), a população da presente pesquisa ainda é pouco investigada na área desenvolvimentista. Observando-se os percentis da avaliação pré-intervenção dos dois grupos investigados, os resultados encontrados sugerem que esta é uma população de risco para atrasos no desenvolvimento.

Um resultado que merece atenção se refere aos bebês do grupo controle terem apresentado diminuição na média da maioria dos escores do momento pré para o pós-intervençáo. Ressalte-se que mesmo que esta diferença não tenha sido significativa, pode ser devastadora para a criança. Outro estudo que avaliou o desenvolvimento motor de bebês internados em unidade de internação pediátrica já vem apontando a hospitalização como um fator de risco para atrasos no desenvolvimento (PANCERI et al., 2012), evidenciando a necessidade interventiva que aqui propomos. Diversos estudos também reportam o período em UTI neonatal como negativo para o desenvolvimento motor de bebês (HALPERN et al., 2000; BLAUW-HOSPERS; HADDERS-ALGRA, 2005; SACCANI et al., 2013). Um estudo reportou que a permanência no hospital e em UTI neonatal repercute negativamente no desenvolvimento motor (SACCANI et al., 2013). Em outra pesquisa, a prevalência de atraso no desenvolvimento neuropsicomotor de 1.363 crianças do sul do Brasil, aos 12 meses de idade, estava associada com a hospitalização das mesmas (HALPERN et al., 2000). Ainda, os procedimentos dolorosos dentro do hospital afetam o desenvolvimento cognitivo e motor de bebês de 8 a 18 meses ( $n=211)$. Funçóes cognitiva e motora são mais baixas com maior número de procedimentos dolorosos, independentemente da gravidade da doença da criança (GRUNAU et al., 2009). Tais resultados, somados ao resultado do grupo controle do presente estudo, demonstram o ambiente hospitalar e as práticas a que o bebê precisa ser submetido em uma internação como possível causa para atrasos no desenvolvimento.

Outro resultado apresentado foi que a interação com fator tempo $\times$ grupo evidenciou diferença significativa nos escores cognitivos, mas não motores avaliados pela Bayley, aceitando, em parte, a segunda hipótese desta pesquisa. A facilitação do desenvolvimento cognitivo através de programas de intervençáo motora já vem sendo discutida na literatura (ALMEIDA; VALENTINI, 2010). O melhor desenvolvimento motor permite que a criança tenha mais oportunidades de interagir com o ambiente, $\mathrm{o}$ que pode facilitar o seu desenvolvimento cognitivo (BLAUW-HOSPERS et al., 2007).

Deve-se considerar o fator limitante do pequeno tamanho amostral da presente pesquisa, o que pode justificar, em parte, a falta de significância nos escores motores. Uma amostra maior pode ser necessária para determinar se um resultado no desenvolvimento motor poderia ser diferenciado. Entretanto, amostras grandes não são possíveis em ambientes hospitalares, a não ser que a pesquisa se estenda por um maior número de anos. Outra possível razão para não se ter encontrado um efeito significativo se refere às variaçóes quanto à quantidade de sessóes, visto que a intervenção aconteceu apenas no período em que o bebê permaneceu no hospital, variando de 4 a 30 dias; note-se que tal limitaçáo se encontra fora do controle dos pesquisadores. Cameron, Maehle e Reid (2005) também reportam dificuldade em encontrar resultados significativos ao avaliarem o 
impacto de um programa de fisioterapia durante a estadia de prematuros na UTI neonatal. As autoras sugerem as mesmas limitaçóes do presente estudo como possíveis causas dos resultados encontrados. Dificuldades como estas estão presentes em pesquisas desenvolvidas dentro do ambiente hospitalar.

Em relação aos escores por posturas da AIMS obtidos nesta pesquisa, as posiçóes prono e supino demonstraram interação significativa no fator grupo $\times$ tempo. Estes resultados diferem dos encontrados em outros estudos com bebês brasileiros, nos quais a postura prono foi apontada com pontuaçóes inferiores em relação aos bebês canadenses (LOPES; LIMA; TUDELLA, 2009; SACCANI; VALENTINI, 2010). Estas duas posturas, prono e supino, no caso de bebês hospitalizados, são aquelas em que eles permanecem mais tempo e, portanto, há mais possibilidades de explorar. A falta de significância nas posiçôes sentado e em pé pode ser explicada pela idade dos bebês. Como mais da metade dos bebês tinha menos de cinco meses, estas posiçôes ainda se encontravam em estágios iniciais. Todas as quatro posiçóes apresentaram diferenças significativas do período pré para o pós-intervenção no GI. Ao analisar separadamente as quatro posturas, diversos estudos encontram resultados diferentes, sugerindo superioridade em algumas posiçóes e não em outras (SACCANI; VALENTINI, 2010; PEREIRA et al., 2011). Esta divergência nos resultados pode ser atribuída à idade das crianças estudadas, assim como às diferentes abordagens interventivas (PEREIRA et al., 2011).

\section{Conclusão}

Os resultados do presente estudo sugerem que a intervenção cognitivo-motora no ambiente hospitalar, durante o tempo de internação de bebês com doenças respiratórias, contribui de forma positiva para o desenvolvimento motor e cognitivo, protegendo esses bebês dos efeitos negativos no desenvolvimento que a hospitalização acarreta. Ressalta-se, assim, a importância de programas de intervençáo dentro do hospital, com o objetivo de oferecer estímulos adequados ao desenvolvimento infantil.

Pesquisas como esta podem servir de alicerce para novos estudos com a população de crianças que precisam de internação hospitalar, após a fase neonatal. Porém, sugere-se maior tamanho amostral e, consequentemente, maior tempo de coleta de dados - para se evitarem limitaçóes como do presente estudo -, assim como novos estudos de follow-up, avaliando estas crianças algum tempo após a alta hospitalar.

\section{Agradecimentos}

Agradecemos, pelo apoio recebido, ao Fundo de Incentivo à Pesquisa e Eventos (FIPE) do Hospital de Clínicas de Porto Alegre (HCPA).

\section{Referências}

ALMEIDA, C. S.; VALENTINI, N. C. Integração de informaçáo e reativação da memória : impacto positivo de uma intervenção cognitivo-motora em bebês. Revista Paulista de Pediatria, São Paulo, v. 28, n. 1, p. 15-22, 2010.

ALMEIDA, K. M. et al. Validade concorrente e confiabilidade da Alberta Infant Motor Scale em lactentes nascidos prematuros. Jornal de Pediatria, Rio de Janeiro, v. 84, n. 5, p. 442-448, 2008.

AMORIM, R. C. A. et al. Programa de saúde da família : proposta para identificação de fatores de risco para o desenvolvimento neuropsicomotor. Brazilian Journal of Physical Therapy, São Carlos, v. 13, n. 6, p. 506-513, 2009.

ARAÚJO, B. B. M.; RODRIGUES, B. M. R. D. Vivências e perspectivas maternas na internação do filho prematuro em Unidade de Tratamento. Revista da Escola de Enfermagem da USP, São Paulo, v. 44, n. 4, p. 865872, 2010.

BAYLEY, N. Bayley scales of infant development. San Antonio: The Psychological Corporation, 1993.

BERSCH, Â. A. S.; YUNES, M. A. M. O brincar e as crianças hospitalizadas: contribuiçôes da abordagem ecológica. Ambiente \& Educação, Rio Grande, v. 13, p. 119-132, 2008

BLAUW-HOSPERS, C. H. et al. Does early intervention in infants at high risk for a developmental motor disorder improve motor and cognitive development? Neuroscience and biobehavioral reviews, New York, v. 31, n. 8, p. 1201-1212, 2007.

BLAUW-HOSPERS, C. H.; HADDERS-ALGRA, M. A systematic review of the effects of early intervention on motor development. Developmental Medicine and Child Neurology, Oxford, v. 47, n. 6, p. 421-432, 2005.

BONNIER, C. Evaluation of early stimulation programs for enhancing brain development. Acta Paediatrica, Oslo, v. 97, n. 7, p. 853-8, 2008.

CAMERON E. C.; MAEHLE, V.; REID J. The effects of an early physical therapy intervention for very preterm, very low birth weight infants: a randomized controlled clinical trial. Pediatric Physical Therapy, Philadelphia, v. 17, n. 2, p. 107-119, 2005.

CAMPOS, D. et al. Concordância entre escalas de triagem e diagnóstico do desenvolvimento motor no sexto mês de vida. Jornal de Pediatria, Rio de Janeiro, v. 82, n. 6, p. 470-474, 2006. 
FERREIRA, A. M.; BERGAMASCO, N. H. P. Análise comportamental de recém-nascidos pré-termos incluídos em um programa de estimulação tátil-cinestésica durante a internação hospitalar. Brazilian Journal of Physical Therapy, São Carlos, v. 14, n. 2, p. 141-148, 2010.

FORMIGA, C. K. M. R.; PEDRAZZANI, E. S.; TUDELLA, E. Desenvolvimento motor de lactentes pré-termo participantes de um programa de intervençáo fisioterapêutica precoce. Brazilian Journal of Physical Therapy, São Carlos, v. 8, n. 3, p. 239-245, 2004.

GABBARD, C.; KREBS, R. Studying environmental influences on motor development in children. The Physical Educator, Indiana, v. 69, n. 2, p. 136-149, 2012.

GABBARD, C.; RODRIGUES, L. P. Testes contemporâneos de avaliaçáo do comportamento motor infantil. In: MOURA-RIBEIRO, M. V.; GONÇALVES, V. M. Neurologia do desenvolvimento da criança. Rio de Janeiro: Revinder, 2006. p. 243-257.

GRAMINHA, S. S. V.; MARTINS, M. A. DE O. Condiçôes adversas na vida de crianças com atraso no desenvolvimento. Medicina, Ribeirão Preto, v. 30, p. 259-267, 1997.

GRANTHAM-MCGREGOR, S. et al. Developmental potential in the first 5 years for children in developing countries. The Lancet, London, v. 369, n. 9555, p. 6070, 2007.

GRUNAU, R. E. et al. Neonatal pain, parenting stress and interaction, in relation to cognitive and motor development at 8 and 18 months in preterm infants. Pain, Washington, v. 143, n. 1-2, p. 138-146, 2009.

HALPERN, R. et al. Fatores de risco para suspeita de atraso no desenvolvimento neuropsicomotor aos 12 meses de vida. Jornal de Pediatria, Rio de Janeiro, v. 76, n. 6, p. 421-428, 2000.

HALPERN, R.; FIGUEIRAS, A. C. M. Influências ambientais na saúde mental da criança. Jornal de Pediatria, Rio de Janeiro, v. 80, n. 2, p. 104-110, 2004. Suplemento.

HOLT, R. L.; MIKATI, M. A. Care for child development: basic science rationale and effects of interventions. Pediatric Neurology, New York, v. 44, n. 4, p. 239-253, 2011.

LOPES, V. B.; LIMA, C. D.; TUDELLA, E. Motor acquisition rate in Brazilian infants. Infant and Child Development, Oxford, v. 18, n. 2, p. 122-132, 2009.

MADASHI, V. Tradução, adaptação transcultural e evidências de validade das Escalas Bayley III de Desenvolvimento Infantil em uma população do município de Barueri, São Paulo. 2012. 85 f. Dissertação (Mestrado em Distúrbios do Desenvolvimento) - Universidade Presbiteriana Mackenzie, São Paulo, 2012.

MARIA-MENGEL, M. R. S.; LINHARES, M. B. M. Fatores de risco para problemas de desenvolvimento in- fantil. Revista Latino-Americana de Enfermagem, Ribeirão Preto, v. 15, p. 837-842, 2007.

MILGROM, J. et al. Early communication in preterm infants following intervention in the NICU. Early Human Development, Amsterdam, v. 89, n. 9, p. 755-762, 2013.

MITRE, R. M. A.; GOMES, R. A promoção do brincar no contexto da hospitalização infantil como ação de saúde. Ciência \& Saúde Coletiva, Rio de Janeiro, v. 9, n. 1, p. 147-154, 2004.

MOTTA, A. B.; ENUMO, S. R. F. Brincar no Hospital: Estratégia de enfrentamento da hospitalização infantil. Psicologia em Estudo, Maringá, v. 9, n. 1, p. 19-28, 2004.

MUNHÓZ, M. A.; ORTIZ, L. C. M. Um estudo da aprendizagem e desenvolvimento de crianças em situação de internação hospitalar. Educação, Santa Maria, v. 1, n. 58, p. 65-83, 2006.

NELSON, M. N. et al. One-year outcome of auditory-tactile-visual-vestibular intervention in the neonatal intensive care unit: effects of severe prematurity and central nervous system injury. Journal of Child Neurology, Thousand Oaks, v. 16, n. 7, p. 493-498, 2001.

NICACIO, S. L. S. M.; RIBEIRO, A. F. Atividade motora e o estado nutricional de lactentes hospitalizados com sibilância recorrente. Pediatria, São Paulo, v. 32, n. 3, p. 184-190, 2010.

OLIVEIRA, R. R.; OLIVEIRA, I. C. S. Os Doutores da Alegria na Unidade de Internação Pediátrica: experiências da equipe de enfermagem. Escola Anna Nery Revista de Enfermagem, Rio de Janeiro, v. 12, n. 2, p. 230-236, 2008.

ORTON, J. et al. Do early intervention programmes improve cognitive and motor outcomes for preterm infants after discharge? A systematic review. Developmental Medicine and Child Neurology, Oxford, v. 51, n. 11, p. 851-859, 2009

PANCERI, C. et al. A influência da hospitalizaçáo no desenvolvimento motor de bebês internados no Hospital de Clínicas de Porto Alegre. Revista HCPA, Porto Alegre, v. 32, n. 2, p. 161-168, 2012.

PEREIRA, K. R. G. et al. Atividades aquáticas para bebês: influência no desenvolvimento motor. Revista da Educação Física/UEM, Maringá, v. 22, n. 2, p. 159-168, 2011.

PIPER, M. C.; DARRAH, J. Motor assessment of the developing infant. Philadelphia W.B: Saunders Company, 1994.

RAMEY, C. T.; RAMEY, S. L. Early intervention and early experience. The American Psychologist, Washington, v. 53, n. 2, p. 109-120, 1998.

RESEGUE, R.; PUCCINI, R. F.; SILVA, E. M. K. Risk factors associated with developmental abnormalities among high-risk children attended at a multidisciplina- 
ry clinic. Sao Paulo Medical Journal, São Paulo, v. 126, n. 1, p. 4-10, 2008.

SACCANI, R. et al. Associations of biological factors and affordances in the home with infant motor development. Pediatrics International, Japáo, v. 55, n. 2, p. 197-203, 2013.

SACCANI, R. Validação da Alberta Infant Motor Scale para aplicação no Brasil: análise do desenvolvimento motor e fatores de risco para o atraso em crianças de 0 a 18 meses. 2009. 123 f. Dissertação (Mestrado em Ciências do Movimento Humano) - Universidade Federal do Rio Grande do Sul, Porto Alegre, 2009.

SACCANI, R.; VALENTINI, N. C. Análise do desenvolvimento motor de crianças de zero a 18 meses de idade: representatividade dos itens da Alberta Infant Motor Scale por faixa etária e postura. Revista Brasileira de Crescimento e Desenvolvimento Humano, São Paulo, v. 20, n. 3, p. 711-722, 2010.

SANTOS-MONTEIRO, J. et al. Estimulação psicossocial e plasticidade cerebral em desnutridos. Revista Brasileira de Saúde Materno Infantil, Recife, v. 2, n. 1, p. 15-22, 2002.

SCHLITTLER, D. X. C. et al. Efeito da intervenção em esteira motorizada na aquisição da marcha independente e desenvolvimento motor em bebês de risco para atraso desenvolvimental. Revista Paulista de Pediatria, São Paulo, v. 29, n. 1, p. 91-99, 2011.

SMITH, S. L. et al. The effect of massage on heart rate variability in preterm infants. Journal of Perinatology, Philadelphia, v. 33, p. 59-64, 2013.
STANDLEY, J. M.; SWEDBERG, O. NICU music therapy: post hoc analysis of an early intervention clinical program. The Arts in Psychotherapy, London, v. 38, p. 36-40, 2011

THOMAS, J. R.; NELSON, J. K.; SILVERMAN, S. J. Métodos de pesquisa em atividade física. Porto Alegre: Artmed, 2012.

ULlENHAG, A.; PERSSON, K.; NYQVIST, K. H. Motor performance in very preterm infants before and after implementation of the newborn individualized developmental care and assessment programme in a neonatal intensive care unit. Acta Paediatrica, Oslo, v. 98, n. 6, p. 947-952, 2009.

VALENTINI, N. C.; SACCANI, R. Brazilian validation of the Alberta Infant Motor Scale. Physical Therapy, Oxford, v. 93, n. 3, p. 440-447, 2012.

VALLADARES, A. C. A.; SILVA, M. T. A arteterapia e a promoção do desenvolvimento infantil no contexto da hospitalização. Revista Gaúcha de Enfermagem, Porto Alegre, v. 32, n. 3, p. 443-450, 2011.

WALKER, S. P. et al. Child development: risk factors for adverse outcomes in developing countries. The Lancet, Londres, v. 369, n. 9556, p. 145-157, 2007.

WALKER, S. P. et al. Inequality in early childhood: risk and protective factors for early child development. The Lancet, Londres, v. 378, n. 9799, p. 1325-1338, 2011.

WILLRICH, A.; AZEVEDO, C. C. F.; FERNANDES, J. O. Desenvolvimento motor na infância : influência dos fatores de risco e programas de intervenção. Revista Neurociências, São Paulo, v. 17, n. 1, p. 51-56, 2009.

\section{Contribuição dos Autores}

Todas as autoras participaram da concepção do texto e da revisão, assim como leram e aprovaram a versão final.

\section{Fonte de Financiamento}

Fundo de Incentivo à Pesquisa e Eventos (FIPE) do Hospital de Clínicas de Porto Alegre (HCPA - suporte financeiro para aquisição de material) e Coordenação de Aperfeiçoamento de Pessoal de Ensino Superior (CAPES - Bolsa de Doutorado). 